\title{
Screening Patterns in Abusive Abdominal Trauma
}

Doriann M. Alcaide Amador ${ }^{1}$, Megan Marine ${ }^{2}$, Boaz Karmazyn²

${ }^{1}$ Indiana University School of Medicine, ${ }^{2}$ Indiana University School of Medicine-Department of

Radiology and Imaging Sciences

Background: Diagnosis of abusive abdominal trauma (AAT) is often clinically occult. Abdominal CT is the preferred method to diagnose abdominal injuries. However, due to risk of ionizing radiation, and cost, it is performed only in selected children. Recently, elevated liver enzymes were used to screen for occult AAT, but its accuracy is unknown due to inconsistent methodology and results.

Objective: To determine the accuracy of elevated liver enzymes (transaminases) in the diagnosis of AAT and if pancreatic enzymes and clinical findings help in patients' selection for abdominal CT.

\section{Methods:}

A retrospective (2011-2020) study on children younger than 3 years suspected of child abuse. The study group included children that had abdominal CT for suspected AAT, while the control group included similar number of children randomized from 5208 children evaluated for child abuse without an abdominal CT. Patients who had an incomplete medical record, were evaluated for cardiac arrest, or had a CT without contrast were excluded.

\section{Results:}

AAT is rare $0.6 \%(30 / 5434)$ in children suspected of child abuse. Transaminases were obtained in $99.1 \%$ of the AAT patients and $55.3 \%$ of the control cases. $93.1 \%(27 / 29)$ patients with abdominal injuries had elevated transaminases. The specificity and sensitivity for the transaminases in detecting positive abdominal CT was $93.3 \%$ and $90.0 \%$, respectively. Only one additional case was identified with elevated pancreatic enzymes and negative transaminases. There was no clinical or imaging findings that could differentiate between patients with negative and positive abdominal CT scans. Based only on elevated transaminases, 11 CT scans need to be performed for a single positive study.

Conclusion/Potential Impact: Transaminases have high sensitivity in predicting AAT. Universal use of transaminases in all children suspected of child abuse may result in 11 CT scans for one positive study. Therefore, more clinical judgement is necessary in selecting patients for CT. 